\title{
Influencia de la producción de nuevo conocimiento y tesis de postgrado en la categorización de los grupos de investigación en Ciencias Sociales: árbol de decisiones aplicado al modelo científico colombiano
}

\author{
José Hernando Ávila-Toscano*, Ivon Romero Pérez**, Eugenio Saavedra Guajardo***, Ailed Marenco-Escuderos* \\ *Corporación Universitaria Reformada, Barranquilla, Colombia \\ Correo-e: javila@unireformada.edu.co | ORCID iD: https://orcid.org/0000-0002-2913-1528 \\ Correo-e: amarenco@unireformada.edu.co | ORCID iD: https://orcid.org/0000-0002-8174-4479 \\ **Universidad Simón Bolívar. Barranquilla, Colombia \\ Correo-e: ivromero@unisimonbolivar.edu.co | ORCID iD: https://orcid.org/0000-0003-0910-1273 \\ ****Universidad Católica del Maule, Talca, Chile \\ Correo-e: esaavedr@ucm | ORCID iD: https://orcid.org/0000-0003-2843-9739
}

Recibido: 12-12-2017; 2a versión: 23-03-2018; Aceptado: 02-04-2018

Cómo citar este artículo/Citation: Ávila-Toscano, J. H.; Romero Pérez, I.; Saavedra Guajardo, E.; Marenco-Escuderos, A. (2018). Influencia de la producción de nuevo conocimiento y tesis de postgrado en la categorización de los grupos de investigación en Ciencias Sociales: árbol de decisiones aplicado al modelo científico colombiano. Revista Española de Documentación Científica, 41 (4): e218. https://doi.org/10.3989/redc.2018.4.1547

Resumen: A través de este estudio se evaluó la productividad de 168 grupos de investigación colombianos en Ciencias Sociales de acuerdo con sus productos de nuevo conocimiento (artículos incluidos en Web of Science, Scopus y otros índices, libros y capítulos de libros) y formación de recursos humanos (tesis de maestría y doctorado). Por medio de un análisis de árboles de clasificación se identificó la influencia de la producción en la categorización de los grupos dentro del modelo científico colombiano. La publicación de libros sigue siendo frecuente en este campo del conocimiento, aunque no resulta significativa para clasificar los grupos; los productos más representativos fueron los artículos incluidos en WoS o Scopus y los trabajos doctorales. Aunque el modelo científico colombiano incluye una variedad de productos, la clasificación de grupos de investigación privilegia la producción en bases internacionales lo cual no es una práctica común en Ciencias Sociales.

Palabras clave: producción científica; tesis doctoral; ciencias sociales; grupos de investigación; artículos.

Influence of the production of new knowledge and postgraduate theses over the categorization of research groups in Social Sciences: a Decision tree applied to the Colombian scientific model

Abstract: This study assess the productivity of 168 Colombian research groups in social sciences, according to their production of new knowledge (articles included in Web of Science, Scopus and other indexes, books and chapters of books) and human capital (master's and doctoral theses). The production influence in the categorization of the groups in the Colombian scientific model was identified throughout an analysis of classification trees. The production of books is still frequent in this field of knowledge, although it does not significantly influence in the categorization; the most representative products were the articles indexed in WoS or Scopus, along with doctoral theses. Even though the Colombian scientific model includes a variety of products, the classification of research groups privileges the production collected in international databases, which is not a common practice in Social Sciences.

Keywords: scientific production; PhD thesis; social sciences; research groups; articles.

Copyright: (c) 2018 CSIC. Este es un artículo de acceso abierto distribuido bajo los términos de la licencia de uso y distribución Creative Commons Reconocimiento 4.0 Internacional (CC BY 4.0). 


\section{INTRODUCCIÓN}

Los estamentos gubernamentales responsables de la formulación de políticas científicas se encuentran receptivos ante la medición de los avances institucionales en materia científica, llevados en gran medida por los estándares internacionales que privilegian la ponderación cuantitativa de la producción académica (Shin y Cummings, 2010). Con el paso del tiempo, la implementación de estos estándares demanda mayores esfuerzos institucionales, reflejados en la necesidad de aplicar ciertos modelos, estrategias e instrumentos de evaluación para medir los factores de calidad de sus actividades de investigación frente al contexto internacional.

En diversos países de Latinoamérica, los gobiernos han venido estableciendo mecanismos de evaluación de los investigadores desde la década de 1980, enfocándose esencialmente en su productividad como elemento determinante para el avance científico y para el sostenimiento de los programas de investigación que promueven las diferentes agencias; la productividad condiciona, además, para acceder a recursos de financiamiento (Vessuri y Sonsiré, 2010).

Sin embargo, la cuantificación de los avances en investigación no siempre resulta sencilla; entre las distintas áreas del conocimiento existen múltiples objetos de estudio abordados con metodologías y perspectivas teóricas diversas, haciéndoles difícilmente equiparables (Ibarra y otros, 2006). Específicamente las Ciencias Sociales no se han nutrido de manera robusta de índices que permitan conocer, a ciencia cierta, los factores y atributos que influyen en la categorización de los grupos de investigación a partir de su producción científica. Colombia no ha sido ajena a esta situación, junto con Costa Rica, El Salvador, Paraguay y Venezuela, constituye uno de los países cuya cantidad de investigadores en Ciencias Sociales representan del 21 al 30\% de todos los científicos del país (Vessuri y Sonsiré, 2010), siendo sin duda una gran cantidad, sin embargo, afronta dificultades relacionadas con la forma de medir la producción y calidad de dichos investigadores organizados en grupos de investigación, puesto que no es sencillo aplicar un modelo único de medición a todas las áreas del conocimiento sin reconocer los elementos y dinámicas particulares de cada quehacer disciplinario (Larivière y otros 2012).

En tal sentido, la medición de productividad de los grupos de investigación puede tornarse relativa, pues no necesariamente el valor superlativo que tradicionalmente se otorga a la producción de artículos en bases de élite mundial como WoS y Scopus, constituye un indicador acertado para cuantificar el nivel de producción y calidad de todas las disciplinas. En Ciencias Sociales, por ejemplo, se reconoce una preferencia de diversas áreas del conocimiento por publicar libros e investigar de forma individual (Hicks, 2004; Nederhof, 2006), o en el caso de la generación de artículos científicos, es más común que se publiquen en revistas incluidas en índices regionales o locales (Archambault y otros 2006; Gantman, 2011; Rosario-Sierra y otros, 2017). Colombia es ejemplo claro de ello, al tratarse de un país donde es habitual que los investigadores empleen revistas incluidas en Scielo como medio de difusión de sus resultados científicos, esto constituye una suerte de estrategia de fortalecimiento regional frente a la producción de conocimiento (Vélez-Cuartas, LucioArias y Leydesdorff, 2016).

Claramente, los grupos de investigación adscritos a universidades y laboratorios o centros científicos independientes, tienen a su cargo la responsabilidad de generar conocimiento dirigido a la resolución de problemas a nivel social, empresarial o industrial (Rueda-Barrios y Rodenes-Adam, 2016), pero la medición de su productividad y la determinación de sus niveles de calidad depende de contar con bases de datos fiables, que integren los diferentes tipos de productos o formas de comunicación científica considerados relevantes según el área de conocimiento, y la articulación entre lo metodológico y lo teórico a nivel regional y global (Vélez-Cuartas y otros, 2014).

En el caso colombiano, la actividad científica se circunscribe mayoritariamente a las universidades (Aldana, 2010). La evidencia nacional señala que, en la medida en que las universidades tienen mejores respuestas institucionales a los cambios en las políticas científicas, más capacidad de adaptación interna, y profesionales con alta formación, desarrollan niveles más significativos de producción científica (Bucheli y otros, 2012) que se concentra en los grupos institucionales de investigación.

En los últimos años se han venido haciendo esfuerzos por consolidar un proceso de medición de estos grupos, tarea que ha sido asumida por el Departamento Nacional de Ciencia, Tecnología e Innovación (Colciencias), en búsqueda de perfeccionar un modelo que permita evaluar a los grupos tomando en cuenta las diferencias y características de los distintos campos de conocimiento. Sin embargo, claramente existe un privilegio por la generación de artículos publicados en revistas indexadas en los índices bibliográficos de alto nivel (WoS y Scopus), que permitan aumentar la visibilidad e impacto de la producción de nuevo conocimiento (Colciencias, 2014). Vale decir, que este modelo no desconoce otra diversidad de productos como son los libros y capítulos de libro de investigación, 
así como productos de divulgación, de apropiación social y de formación de recursos humanos, estos últimos referidos a tesis de grado a nivel de maestría y doctorado (Colciencias, 2017).

Este modelo de medición es un instrumento creado con el propósito de conocer las capacidades y dinámicas de los grupos de investigación de Colombia, con miras a fortalecer la gestión y la toma de decisiones frente a las políticas de estímulo y priorización de los recursos de inversión, financiación y sostenibilidad de los proyectos de CTeI. Este modelo conceptual, matemático y estadístico describe la categorización de los grupos de investigación en diversos niveles; inicialmente eran cinco, denominados categorías ( 1 1, A, B, C y D), cuya vigencia perduró de 2012 hasta 2016, pero a partir de 2017, con los ajustes que viene sufriendo el modelo con la finalidad de robustecerlo, se suprimió la última categoría.

La categorización se obtiene a partir de la distribución en cuartiles de la productividad global de los grupos por área de conocimiento. Esta clasificación parte del cálculo de indicadores de producción asociados con los índices de colaboración, cohesión, circulación, producción de nuevo conocimiento, desarrollo tecnológico, apropiación social y formación de recursos humanos (Colciencias, 2017).

Una vez implementado entre los años 2013 al 2015, y luego de analizarse el informe de 2016 realizado por el Observatorio Colombiano de Ciencia y Tecnología (OCYT), puede inferirse un efecto positivo del modelo en el incremento del índice de nuevo conocimiento en Ciencias Sociales, especialmente si se analizan los años 2014 y 2015 que muestran un incremento del $3.83 \%$ y $1.47 \%$ respectivamente, de la producción colombiana en revistas indexadas en WoS y Scopus (OCyT, 2016). Sin embargo, estos datos contrastan con los indicadores de productividad global (todas las áreas) presentado por la Red de Indicadores de Ciencia y Tecnología Iberoamericana e Interamericana (RICYT) en el 2016, debido a que sigue manteniéndose un rezago en la productividad de Colombia en comparación con países latinoamericanos como Brasil, México, Argentina y Chile (RICYT, 2016).

Sin embargo, como ya se ha dicho, la medición de la productividad científica no se restringe a la publicación de artículos en bases internacionales, sino que contempla diversas categorías en las que se reconoce incluso el trabajo de formación para investigadores (tesis) a nivel de postgrado, la articulación con organizaciones sociales y procesos de apropiación, y la generación de otros productos de nuevo conocimiento como los libros y capítulos de libros, lo que constituye a la luz de algunos autores, mecanismos de vanguardia en la evaluación de grupos de investigación (Vélez-Cuartas y otros, 2014).

En el caso de las tesis de postgrado, ha crecido el interés por su análisis, pasando a ser consideradas como un indicador importante en la capacidad de formación investigativa de las universidades (Sanz-Casado y otros, 2011), además de aportar significativamente al estudio de fenómenos sociales relevantes, con logros científicos valiosos para los investigadores, las disciplinas y los países mismos (Fernández-Cano y otros, 2011), incluso, la producción de trabajos doctorales se ha asociado con el aumento de la publicación de artículos incluidos en WoS y Scopus (Sánchez y otros, 2017), en cuanto muchos de estos se derivan de la investigación doctoral.

También se ha reseñado el valor de las dinámicas de citación en tesis de maestría y doctorado para identificar los patrones de uso del material científico, los cuales varían de acuerdo con las disciplinas (Becker y Chiware, 2015; Rosenberg, 2015). Ahora bien, los libros y capítulos de libros resultado de la investigación, también son sustanciales al momento de evaluar la producción de grupos en Ciencias Sociales, pues constituyen el medio más tradicionalmente empleado para comunicar el conocimiento en estas disciplinas (Rosario-Sierra y otros, 2017), de forma que no incluirlos afecta la visibilidad e impacto de los hallazgos en los circuitos de reconocimiento internacional para las Ciencias Sociales.

De aquí la necesidad de realizar el presente estudio, en el cual se han trazado dos objetivos principales, el primero consiste en evaluar la productividad de grupos de investigación en el campo de las Ciencias Sociales, contemplando en este caso el análisis de la producción de nuevo conocimiento indexada en WoS y Scopus, pero también en otros índices locales y regionales (Publindex, Redalyc, Scielo, LILACS, etc.), así como otros productos científicos (libros y capítulos de libros) y de formación de recursos humanos (tesis). El segundo objetivo de este trabajo consiste en probar un modelo de clasificación a través de un análisis multivariado basado en técnicas bibliométricas y estadísticas, dirigido a reconocer el peso de la producción de los grupos para alcanzar categorías significativas dentro del modelo de medición colombiano, que intenta ofrecer alternativas diferenciales a nivel disciplinar.

\section{MÉTODO}

\subsection{Diseño y unidad de análisis}

Se desarrolló un estudio bibliométrico con corte retrospectivo, a través del cual se evaluó la pro- 
ducción de 168 grupos de investigación de Ciencias Sociales, clasificados en el Sistema Nacional de Ciencia, Tecnología e Innovación (ScienTI) de Colombia según los resultados de la convocatoria de 2015, vigente hasta septiembre de 2017.

Se analizó la producción del periodo 2006-2015 generada por grupos de las subáreas Educación, Ciencias Políticas, Derecho, Psicología, Sociología, Periodismo y otras Ciencias Sociales (Antropología, Economía y otros). La selección de estas subáreas obedece a la clasificación dentro del ScienTI Colombia, el cual ha sido ajustado para guardar equivalencias con la clasificación de la Organización para la Cooperación y el Desarrollo Económico (OCDE) (Colciencias, 2015). Estas subáreas sumaban 1019 grupos, sin embargo, el análisis se centró en los grupos con mayor clasificación (categorías A1, A y B) los cuales sumaban un total de 375 (Colciencias, 2015); se excluyeron, por tanto, los grupos categoría C y D. De acuerdo con el Modelo de medición (Colciencias, 2015), los criterios para obtener las categorías en mención se describen en la Tabla I.

La selección de los grupos fue realizada por muestreo por estratos con afijación proporcional para muestras finitas, definiendo un nivel de confianza del 95\%. Dado que los grupos con categoría A1 no son numerosos, se decidió incluir todos los grupos A1 como parte de la muestra, mientras que los grupos categoría A y B se estratificaron. La Tabla II contiene la descripción de todo el proceso de muestreo hasta completar el número final de grupos.

Tabla I. Requisitos para la clasificación de grupos de investigación colombianos según el Modelo de Medición 2015 de Colciencias

\begin{tabular}{|c|c|c|c|}
\hline & \multicolumn{3}{|c|}{ Categoría del grupo } \\
\hline Criterios de clasificación & $\mathrm{A} 1$ & A & B \\
\hline Indicador de grupo ${ }^{a}$ & Cuartil 1 & Cuartil 2 & Cuartil 3 \\
\hline Productos TOP o productos $A^{b}$ & Cuartil 1 & $>0$ & $>0$ \\
\hline Productos de apropiación social & $>0$ & $>0$ & $>0$ \\
\hline Formación de Recursos Humanos ${ }^{c}$ & Tipo $A>0$ & Tipo $A>0$ & Tipo A $>0$, o Tipo B Cuartil2 \\
\hline Tener investigadores clasificados ${ }^{d}$ & SoA & SoA & $S, A, J \circ P h D$. \\
\hline Indicador de cohesióne & $>0$ & $>0$ & $>0$ \\
\hline Tiempo de existencia & 5 años & 5 años & 3 años \\
\hline
\end{tabular}

a Valor derivado de la suma de todos los indicadores de cada grupo; ${ }^{b}$ Cuartiles 1 y 2 de WoS o Scopus; ${ }^{c}$ Tipo A= Tesis y programas de doctorado, Tipo $B=$ Tesis y programas de maestría, trabajos de pregrado y otros; ${ }^{d} \mathrm{~S}=$ Sénior, $\mathrm{A}=\mathrm{Asociado}, \mathrm{J}=\mathrm{Junior}$, $\mathrm{PhD}=$ Integrante con doctorado; ${ }^{\mathrm{e}}$ Colaboración interna entre integrantes del grupo.

Tabla II. Muestreo por estratos con afijación proporcional

\begin{tabular}{|l|c|c|c|c|c|c|c|c|c|}
\hline \multirow{2}{*}{ Áreas del conocimiento } & \multirow{2}{*}{$\mathbf{N}$} & \multirow{2}{*}{$\mathbf{n}$} & \multicolumn{4}{|c|}{$\mathbf{n i}$} & \multicolumn{2}{c|}{ Muestra del estrato } & \multirow{2}{*}{ Total } \\
\cline { 5 - 11 } & & & A1 & A & B & A1 & A2 (\%) & B (\%) & \\
\hline Educación & 343 & 135 & 9 & 61 & 65 & 9 & $24(17.8)$ & $26(19)$ & 59 \\
\hline Psicología & 144 & 63 & 13 & 19 & 31 & 13 & $8(13.2)$ & $14(21.5)$ & 35 \\
\hline Derecho & 193 & 56 & 4 & 16 & 36 & 4 & $6(8.3)$ & $10(18.7)$ & 20 \\
\hline Otras Ciencias Sociales & 131 & 50 & 2 & 13 & 35 & 2 & $5(9.9)$ & $13(26.7)$ & 20 \\
\hline Ciencias Políticas & 64 & 29 & 7 & 9 & 13 & 7 & $4(14.1)$ & $6(20.3)$ & 17 \\
\hline Periodismo & 69 & 22 & 3 & 3 & 16 & 3 & $1(4.3)$ & $5(23.2)$ & 9 \\
\hline Sociología & 75 & 20 & 3 & 6 & 11 & 3 & $2(8)$ & $3(14.7)$ & 8 \\
\hline Totales & 1019 & 375 & 41 & 127 & 207 & 41 & 50 & 77 & 168 \\
\hline
\end{tabular}

$\mathrm{N}=$ total grupos por subárea; $\mathrm{n}=$ grupos $\mathrm{A} 1$, $\mathrm{A}$ y $\mathrm{B}$ por subárea; $\mathrm{n}$ i=número de grupos en cada estrato. 


\subsection{Variables de estudio}

Se consideraron dos conjuntos de variables de acuerdo con el tipo de producción clasificado por ScienTI Colombia: los Productos de Nuevo Conocimiento y los Productos de Formación de Recursos Humanos.

Productos de Nuevo Conocimiento. Aportes significativos a un área del conocimiento derivados de investigación, desarrollo tecnológico o innovación. Para el caso de este estudio se incluye:

a) Artículos científicos: Se subdividen según su nivel de impacto. Por un lado, están los artículos publicados en revistas indexadas en los índices bibliográficos de citaciones ISI -Web of Knowledge (Science Citation Index [SCI] y Social Science Citation Index [SSCI]) o en Scopus. El nivel de importancia se ajusta al cuartil en el que se encuentre la revista Q1, Q2, Q3 o Q4, de modo que se les categoriza como $A 1, A, B$ o $C$ respectivamente.

Además, se encuentran los artículos Tipo $D$, que corresponden a publicaciones en revistas que no están indexadas en WoS o Scopus pero que están incluidas en dos o más Bases Bibliográficas con Comité Científico (BBCC) reconocidas por ScienTI Colombia (Colciencias, 2015), por ejemplo: Scielo, LILACS, Social Science Abstracts, etc.

Para efectos prácticos en este estudio se les denominará artículos WoS/Scopus y artículos tipo D.

b) Libros resultados de investigación: libro completo que difunde resultados inéditos y originales de investigación que han sido evaluados por pares de forma anónima. Posee requisitos mínimos de calidad en cuanto a catalogación y edición.

c) Capítulos de libros resultados de investigación: publicaciones inéditas, originales, que divulgan resultados de investigación, y que forman parte de un libro de colaboración conjunta.

Productos de Formación de Recursos Humanos. Productos derivados de la formación de nuevos investigadores o recursos humanos para la ciencia, tecnología e innovación. En este estudio se han considerado las Tesis de Doctorado y las Tesis de Maestría, es decir, los productos de formación de alto nivel. Se excluyeron los trabajos de pregrado.

\subsection{Procedimiento y análisis de datos}

Este proyecto se desarrolló bajo el acompañamiento del Departamento Administrativo de Ciencia, Tecnología e Innovación de Colombia (Colciencias), organismo financiador y supervisor. La información requerida para el análisis de los datos se obtuvo de la plataforma tecnológica ScienTI, la cual es de acceso público y permite la consulta de la producción de los grupos de investigación clasificados en las diferentes subáreas consideradas en este estudio.

El registro de todos los artículos se realizó contrastando la información incluida en el reporte de cada grupo de investigación, de esta forma, todos los artículos reportados, independientemente de su categoría, fueron consultados de forma directa en la respectiva revista reportada en ScienTI. Esto permitió garantizar la fidelidad de la información y prevenir la existencia de registros erróneos. En el caso de los artículos publicados en revistas WoS y Scopus, se depuró la basé de datos manualmente para evitar la doble contabilización de los artículos de revistas que estuvieran incluidas en ambas bases de datos. Todos los registros se cumplieron entre diciembre de 2016 y junio de 2017.

Los datos fueron sometidos al respectivo análisis descriptivo y posteriormente se realizó análisis comparativo mediante pruebas no paramétricas, para ello, la cantidad de productos de nuevo conocimiento y de formación de recursos humanos fue comparada según la categoría de los grupos de investigación con la prueba $\mathrm{H}$ de Kruskal-Wallis, dado que las variables no cumplieron con el supuesto de normalidad (Komogorov-Smirnov $p=0,05$ ). El pos hoc se cumplió con la U de Mann-Whitney, calculando el tamaño de efecto con la $r$ de Rosenthal.

También se evaluaron las variables que determinan la clasificación de los grupos de investigación. Este proceso se cumplió a través del algoritmo Classification and Regression Trees (CART) (Breiman y otros, 1984) que permite crear árboles de decisión. El procedimiento CART se basa en análisis no paramétrico y facilita el trabajo con variables independientes o dependientes continuas y categóricas, lo que ofrece una ventaja sobre otros algoritmos como CHAID (Chi-square Automated Interaction Detection), por ejemplo, que realiza la función de clasificación sobre la base de independientes categóricas.

El procedimiento CART consiste en realizar un análisis de partición de variables seleccionando las que son más relevantes dentro de un modelo de regresión (Low y otros, 2014; Steinberg, 2009). A partir de una variable central (dependiente) denominada nodo padre, el algoritmo identifica las variables predictoras (independientes) significativas en orden de relevancia. La función se basa en la partición de la variable dando como resultado dos nodos hijos (Daud y otros, 2015), esta división se realiza con base en la mayor homogeneidad de los dos conjuntos de datos, de esta forma, si se elige 
la variable $x_{j}$, entonces la muestra se divide en $x_{j} \leq$ c y $x_{j}>c$. Este procedimiento es iterativo, de modo que se aplica a cada subgrupo (nodo) resultante de la partición empleando otra variable predictora, hasta que no se hallen nuevas particiones que resulten significativas. CART incluye una función de poda (pruning) que permite eliminar hojas y ramas del árbol, con el fin de prescindir de ramas en las cuales la división se basa en pocos datos lo que afectaría la predicción. Esto garantiza el tamaño óptimo del árbol con las divisiones más confiables y que más aportan al análisis.

\section{RESULTADOS}

\subsection{Productos de Nuevo Conocimiento}

\section{Artículos incluidos en WoS/Scopus y otras Bases bibliográficas con Comité Científico (BBCC)}

Durante el periodo de estudio (2006-2015) los grupos de investigación colombianos publicaron un total de 2992 artículos en revistas científicas $(\mu=299,2 ;$ de=56,5; Mín.=208, Máx.=377); 42,7\% de estos productos $(n=1279)$ fueron obtenidos durante el periodo 2006-2009, y 57,3\% $(n=1713)$ entre 2011 y 2015, presentándose un aumento promedio de 86,8 contribuciones publicadas durante cada año del segundo quinquenio en relación con el anterior, aunque la comparación entre ambos periodos no tuvo diferencias estadísticamente significativas $\left(U_{[2992]}=1079862, p=, 667>, 05\right)$.

Del total de artículos, 1341 (44,8\%) fueron publicados en revistas incluidas en las bases de WoS o Scopus $(\mu=7,98 ;$ de $=10,3$; Mín.=0, Máx.=48), mientras que $1651(55,2 \%)$ fueron productos tipo D $(\mu=9,8 ; d e=11,5 ;$ Mín.=0, Máx.=76). Vale decir que en esta cantidad de artículos tipo $D$ están incluidas 115 producciones publicadas en revistas que al momento del estudio formaban parte de WoS o Scopus, pero que no contaban con esta categorización cuando los documentos fueron publicados.

Los grupos categoría A1 sobresalieron con la mayor cantidad de artículos publicados $(n=1361$; $45,5 \%)$, seguidos de los de categoría B $(n=860$; $28,7 \%)$ y en tercer lugar los de categoría A ( $n=771$; $25,8 \%$ ), esta diferencia es estadísticamente significativa según lo expresa el estadístico de KruskalWallis $\left(x^{2}{ }^{2}=40,356, p=, 000<, 05\right)$. Este orden de producción también se mantuvo para los productos incluidos en WoS y Scopus ( $A 1=749[55,9 \%]$; $\mathrm{A}=288 \quad[21,5 \%] ; \mathrm{B}=304[22,7 \%]) \quad\left(x^{2}{ }_{[2]}=42,370\right.$, $p=, 000<, 05)$ y para los artículos tipo $\mathrm{D}$ (A1=612[37,1\%]; $A=483[29,3 \%] ; B=556[33,7 \%])$ $\left(x^{2}{ }_{[2]}=17,250, p=, 001<, 05\right)$. En la Tabla III se describen todos los contrastes post hoc con su correspondiente tamaño de efecto para cada una de las variables descritas.

\section{Libros y capítulos de libros resultado de investigación}

Los 168 grupos evaluados reportaron la publicación de 1587 libros resultados de investigación, con una media de 9.4 producciones $(d e=8,7 ;$ Mín. $=0$, Máx. $=42)$; el $90 \%(n=1428)$ de esta producción ha sido publicada en Colombia, y la restante en otros 20 países dentro de los que sobresalen España, Argentina, Francia y Alemania, que acumulan el $7,5 \%$ de los textos.

Tabla III. Prueba U de Mann Whitney con su respectivo tamaño de efecto para los artículos publicados según grupo de investigación

\begin{tabular}{|c|c|c|c|c|c|c|c|}
\hline Variable & Grupo 1 & $\begin{array}{c}\text { Grupo } \\
\text { contraste }\end{array}$ & $\boldsymbol{U}$ & $z$ & $p$ & $\boldsymbol{r}^{a}$ & $>R M$ \\
\hline \multirow{3}{*}{ Total artículos publicados } & Grupo A1 & A & 527 & -3.793 & $.000 *$ & $.40^{i}$ & $\mathrm{~A} 1$ \\
\hline & & $B$ & 519 & -5.989 & $.000 *$ & $.55^{9}$ & $\mathrm{~A} 1$ \\
\hline & Grupo A & B & 1459 & -2.300 & $.021 *$ & $.20^{\mathrm{p}}$ & A \\
\hline \multirow[t]{3}{*}{ Artículos WoS-Scopus } & Grupo A1 & A & 439.5 & -4.678 & $.000 *$ & $.49^{i}$ & $\mathrm{~A} 1$ \\
\hline & & $B$ & 483.5 & -6.218 & $.000 *$ & $.57^{9}$ & $\mathrm{~A} 1$ \\
\hline & Grupo A & $B$ & 1502 & -2.106 & $.035 *$ & $.19^{p}$ & A \\
\hline \multirow[t]{3}{*}{ Artículos tipo D } & Grupo A1 & A & 643.5 & -3.049 & $.002 *$ & $.32^{i}$ & $\mathrm{~A} 1$ \\
\hline & & $B$ & 857.5 & -4.082 & $.000 *$ & $.38^{i}$ & $\mathrm{~A} 1$ \\
\hline & Grupo A & $\mathrm{B}$ & 1768.5 & -0.775 & .439 & - & - \\
\hline
\end{tabular}

$\mathrm{U}=\mathrm{U}$ de Mann Whitney; ${ }^{*} \mathrm{p}<.05 ;{ }^{\mathrm{a}} \mathrm{r}$ de Rosenthal (tamaño de efecto); ${ }^{\mathrm{s}}$ efecto pequeño; ${ }^{\mathrm{I}}$ Efecto Intermedio; ' Efecto grande; n $\operatorname{Sin}$ efecto; $<\mathrm{RM}=$ Grupo con mayor rango medio. 
La cantidad de libros producidos fue concordante con el nivel de clasificación de los grupos siendo un producto más frecuente entre los que tiene mayor clasificación, de esta manera, en la categoría A1 se acumularon 713 libros (44,9\%), un número bastante mayor que el de los grupos categoría A con $469(29,6 \%)$, cuya cantidad de publicaciones fue cercana a la obtenida por los grupos categoría B con $405(22,5 \%)$.

La cantidad de libros publicados por año mostró regularidad oscilando entre 130 y 197 . Al comparar la producción entre los dos quinquenios se observó que en el primero de ellos (2006-2010) fueron publicados 753 libros, y en el segundo (2011-2015) la cifra fue de 834 siendo cercana al periodo inmediatamente anterior, de hecho, las diferencias de medias para ambos periodos no fueron significativas $\left(U_{[1587]}=298061, p=, 08>, 05\right)$.

Por su parte, los capítulos de libros de investigación registrados fueron $1737(\mu=10,3$; de=14,3; Mín.=0, Máx.=84); $769(44,3 \%)$ desarrollados por grupos categoría A1, $367(21,1 \%)$ por grupos $A, y$ 601 (34,6\%) por grupos B. Los grupos de investigación mostraron diferencias significativas tanto en los libros publicados $\left(x_{[2]}^{2}=15,014, p=, 001<, 05\right)$, como en los capítulos $\left(x^{2}{ }_{[2]}=13,674, p=, 000<, 05\right)$. Como se aprecia en la Tabla IV, estas diferencias se dieron entre los grupos $\mathrm{A} 1$ en relación con los $\mathrm{A}$ y $\mathrm{B}$, mientras que entre estos últimos no hubo diferencias.

\section{Productos de Formación de Recursos Humanos}

Tras revisar las plataformas de cada uno de los grupos se identificaron un total de 4487 tesis de culminación de estudios de postgrado de alto nivel. De esa cifra la mayoría correspondió a Tesis de maestría (TesisMg), siendo en total 4213 producciones $(\mu=24,42, d e=26,7 ;$ Mín.=0, Máx.=163). Los restantes 274 productos correspondieron a Tesis de doctorado (TesisPhD) $(\mu=1,64$, de $=3,53$; Mín. = 0, Máx.=26).
Con el paso de los años se ha dado un aumento importante de la cantidad de productos de formación generados por los grupos de investigación en Ciencias Sociales (ver Figura 1), pasando de 117 TesisMg en 2006, a 605 en 2015, y un tope de 700 en 2014. El promedio de TesisMg en el primer quinquenio fue de 242 por año mientras que en el segundo fue de 600 , lo que demuestra este aumento importante con el paso del tiempo.

Para las TesisPhD ocurre algo similar, aunque claramente la cantidad es considerablemente menor frente a la formación en maestría. En 2006 el número de TesisPhD reportado fue de 12 , mientras que en 2015 fue de 45, siendo 2012 el año de más tesis de este nivel con 51 . Como sucede con las TesisMg, el promedio de trabajos concluidos en el primer quinquenio es menor en comparación con los últimos cinco años del periodo evaluado, pasando de 12,2 trabajos anuales a 42,6.

\subsection{Análisis multivariado}

Con el fin de identificar cuáles son los productos que pronostican la clasificación de los grupos analizados, se aplicó la técnica de árboles de decisión con el método de crecimiento Classification and Regression Trees (CART).

El árbol de decisión se dibujó empleando la clasificación de los grupos como variable dependiente, mientras que las variables independientes incluidas fueron los productos de nuevo conocimiento y los productos de formación. Para el nodo parental se definió en 100 el número de casos mínimos, y en 50 para cada nodo filial. El resultado fue un árbol de 5 nodos, con 3 nodos terminales y una profundidad de 2 como se aprecia en la Figura 2. Este procedimiento pronostica correctamente el $65.5 \%$ (Riesgo $=34,5 \%$ ) de los grupos, siendo el más correcto los de categoría $\mathrm{B}(83,1 \%)$, mientras que las dos categorías restantes tienen niveles muy discretos $(A 1=41,5 \% ; A=51 \%)$.

Tabla IV. Prueba U de Mann Whitney con su respectivo tamaño de efecto para los libros y capítulos de libros publicados según grupo de investigación

\begin{tabular}{|l|c|c|c|c|c|c|c|}
\hline Variable & Grupo 1 & $\begin{array}{c}\text { Grupo } \\
\text { contraste }\end{array}$ & $\boldsymbol{U}$ & $\boldsymbol{Z}$ & $\boldsymbol{p}$ & ra & $>\boldsymbol{R M}$ \\
\hline \multirow{2}{*}{ Libros de investigación publicados } & \multirow{2}{*}{ Grupo A1 } & $\mathrm{A}$ & 776,000 & $-1,989$ &, $047^{*}$ &, $21^{\mathrm{p}}$ & $\mathrm{A} 1$ \\
\cline { 3 - 8 } & & $\mathrm{B}$ & 914,000 & $-3,764$ &, $000^{*}$ &, $35^{\mathrm{i}}$ & $\mathrm{A} 1$ \\
\hline $\begin{array}{l}\text { Capítulos de libro de investigación } \\
\text { publicados }\end{array}$ & \multirow{2}{*}{ Grupo A1 } & $\mathrm{A}$ & 650,000 & $-2,996$ &, $003^{*}$ &, $31^{\mathrm{i}}$ & $\mathrm{A} 1$ \\
\cline { 3 - 8 } & & $\mathrm{B}$ & 963,500 & $-3,484$ &, $000^{*}$ &, $32^{\mathrm{i}}$ & $\mathrm{A} 1$ \\
\hline
\end{tabular}

$\mathrm{U}=\mathrm{U}$ de Mann Whitney; ${ }^{*} \mathrm{p}<.05 ;{ }^{\mathrm{a}} \mathrm{r}$ de Rosenthal (tamaño de efecto); ${ }^{\mathrm{s}}$ efecto pequeño; ${ }^{\mathrm{I}}$ Efecto Intermedio; 'Efecto grande; n $\mathrm{Sin}$ efecto; $<\mathrm{RM}=$ Grupo con mayor rango medio. 
Figura 1. Distribución de productos según su nivel de formación por año

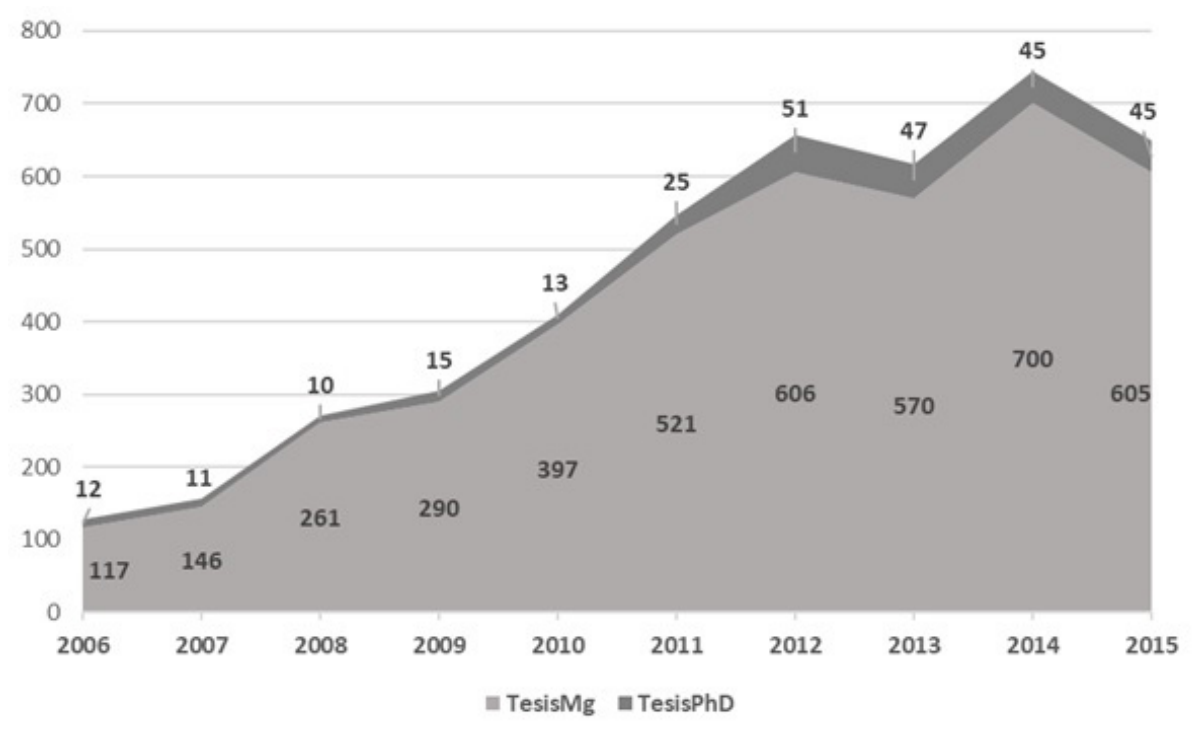

Figura 2. Árbol de decisión para la variable Clasificación de los grupos

Clasificación del grupo

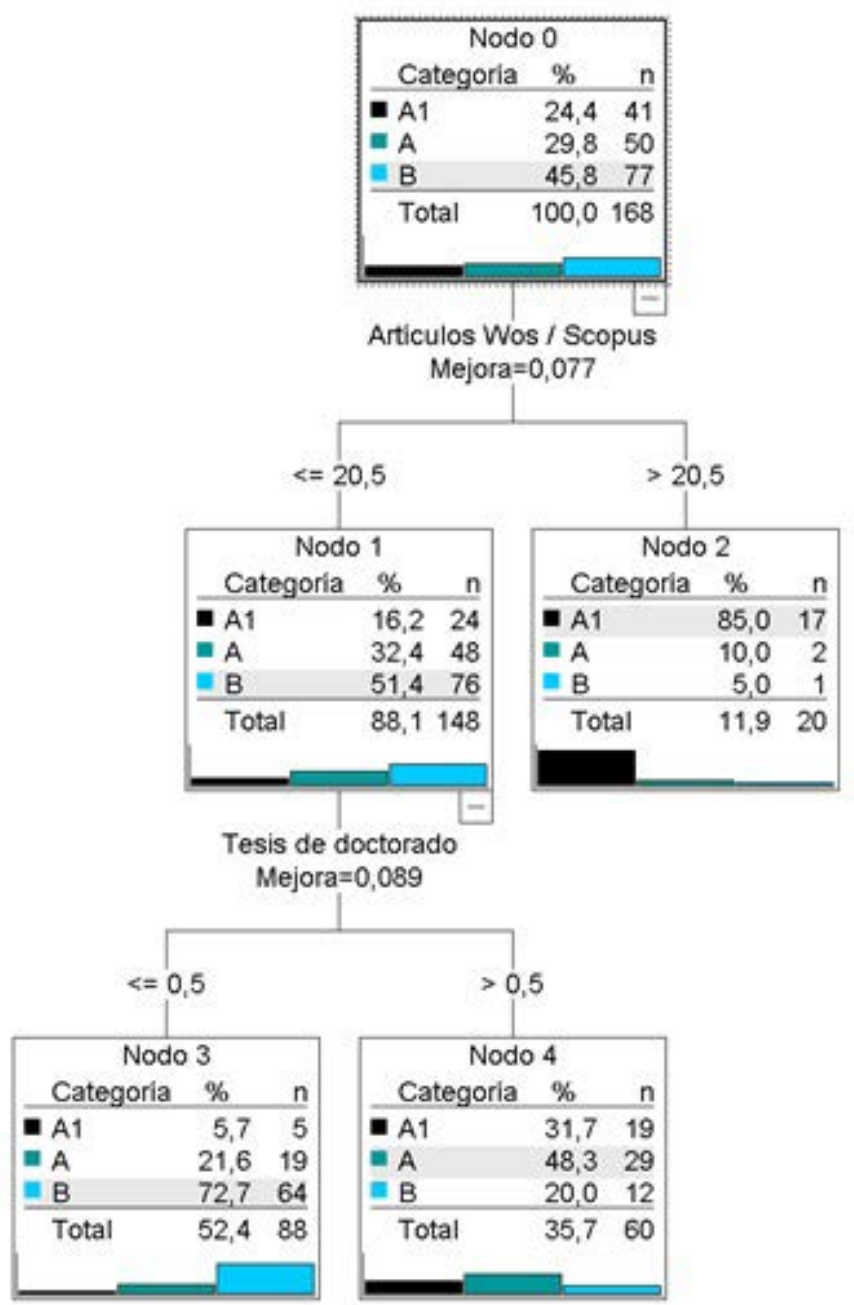


De acuerdo con este modelo, se descartó la participación de las tesis de maestría, libros y capítulos de libros, por no ofrecer predicciones significativas de la clasificación de los grupos de investigación, en cambio, los datos señalan que son dos los productos que pronostican la categoría de los grupos, por un lado, en los grupos con elevada producción de artículos incluidos en WoS/Scopus $(>=20,5)$ el $11,9 \%$ de los casos son clasificados en categoría A1, mientras que una producción por debajo del valor de segmentación $(<20,5)$ pronostica la clasificación en categoría $\mathrm{B}$ en el $88,1 \%$ de los casos. Adicionalmente, en el $52,4 \%$ de los casos, los grupos B además de tener baja cantidad de este tipo de artículos también cuentan con pocas tesis de doctorado $(<=, 5)$. Por último, en los grupos sin elevada producción en WoS/Scopus pero que cuentan con un número importante de Tesis de doctorado $(>, 5)$, el $35,7 \%$ de los casos son pronosticados en categoría A.

Según los resultados del modelo, la variable más importante en la clasificación de los grupos corresponde con artículos incluidos en WoS/Scopus con una importancia de ,100 (100\%), mientras que las tesis doctorales obtuvieron una importancia de ,089 $(88,2 \%)$. Entre tanto, la variable menos relevante fue de artículos tipo D con un valor de ,007 (7\%).

\section{DISCUSIÓN}

¿Cuáles son los productos o formatos de divulgación del conocimiento más pertinentes en la evaluación del estado de la ciencia en las disciplinas sociales? ¿Cómo se puede evaluar a los investigadores sociales y a sus grupos de investigación? Estas son preguntas resonantes y reiteradas en la investigación que aborda la productividad científica de las disciplinas sociales, cuestiones que también son aplicables para las humanidades.

En este estudio se abordaron estos interrogantes a partir del desarrollo de dos objetivos esenciales, el primero, enfocado en evaluar la producción de los grupos de Ciencias Sociales en Colombia, considerando dos conjuntos de productos, los de Nuevo Conocimiento y los de Formación de recursos humanos. El segundo objetivo fijó su interés en identificar cuáles de esos productos mostraban un valor significativo en la clasificación de los grupos de investigación.

En un principio, es necesario recalcar que la producción científica colombiana en Ciencias Sociales no es voluminosa, lo que refuerza evidencias de informes previos en los cuales sobresale el rezago de la productividad de científicos nacionales en comparación con otros países de la región como Brasil, Argentina, México y Chile (RICYT, 2016). Los promedios de producción por grupo de investigación a lo largo del decenio evaluado son tan solo de 7.98 artículos WoS/Scopus, 9.8 artículos tipo D, 9.4 libros y 10.3 capítulos de libro.

En relación con los artículos científicos, se ha venido cerrando la brecha en la producción en índices de alto impacto frente a aquellos de más reconocimiento regional; la publicación de artículos en WoS y Scopus ha venido aumentando, lo que responde a un sistema de medición que estimula mucho más este tipo de producto. Aún con esto, sigue siendo mayor la publicación de artículos en otras bases de indexación (Scielo, Redalyc, LILACS, etc.); ciertamente, las revistas incluidas en estas bases cuentan con formatos más flexibles, incluso con mayor apertura a propuestas metodológicas cualitativas y con líneas editoriales que se ajustan al idioma de los autores (Gantman, 2011), además de ello, se trata de órganos de difusión en los que se generan estrategias de integración sur-sur (Vélez-Cuartas y otros, 2016), lo que garantiza la participación de coautorías entre investigadores con horizontes comunes.

A todo ello se suma el hecho que la mayor parte de las revistas con alto nivel de impacto incluidas en WoS y Scopus son editadas en países angloparlantes (Ibarra y otros, 2006), esto, además de plantear limitaciones idiomáticas para el autor hispano, también conlleva que las líneas temáticas y el público al que se dirige pueden no estar familiarizado con los fenómenos sociales del contexto colombiano, pues como se ha descrito en la literatura especializada, la investigación en Ciencias Sociales tiende a enfocarse en problemas de orden local o regional, con fuertes influencias culturales (Huang y Lin, 2011), de ahí también la predilección por publicar en revistas latinoamericanas (Gantman, 2011), en las que hay más facilidad de compartir intereses sociales.

El libro y los capítulos de libros siguen siendo uno de los formatos apetecibles en las disciplinas sociales; en grupos colombianos se registra un total de 1587 y 1737 productos respectivamente, en el periodo de tiempo evaluado, sin embargo, llama la atención la falta de aporte de estos productos en el modelo de clasificación y regresión, lo que parece sugerir que no ofrecen el suficiente peso para contribuir con la categorización de los grupos de investigación. Un elemento a considerar en el impacto de los libros es su nivel de internacionalización, como es obvio, la publicación internacional ayuda a inscribir los resultados científicos en círculos más amplios de discusión académica (Verleysen y Engels, 2014), sin embargo, esto también implica que los investigadores escojan adecuadamente los escenarios geográficos para lograr visibilidad transfronteriza considerando la relevancia de los temas de estudio, el abordaje de fenómenos que superen el interés local y las características del público lector (Thompson, 2005). 
Ahora bien, en el modelo científico colombiano no existen mecanismos suficientes de control para la calidad de los libros y capítulos de libros; su existencia obedece al registro completo de indicadores de catalogación bibliotecológica en la plataforma digital de los grupos de investigación, y su reconocimiento se ajusta a la presentación de soportes editoriales donde se declara que se trata de trabajos de investigación con revisores externos. A diferencia de los artículos, no hay bases de datos específicas que faciliten revisar que las condiciones de desarrollo de estos productos se cumplan cabalmente. Además, la producción de libros en Colombia es un fenómeno eminentemente doméstico, en su gran mayoría generado a través de los sellos editoriales de las universidades, a esto se suma la baja tasa de citación internacional de este tipo de trabajos, de manera que su reconocimiento como producto científico requiere también del desarrollo de esfuerzos metodológicos dirigidos a generar indicadores específicos que permitan determinar aspectos de calidad editorial susceptibles de aumentar el impacto de estas publicaciones.

Elementos como el nivel de especialización, la garantía de los procesos de calidad editorial mediante revisión por pares, la inclusión de los textos en bases bibliográficas con reconocimiento internacional y hasta contar con adecuados sistemas de posicionamiento en el mercado, son factores que determinan en gran medida el nivel de impacto de los libros (Giménez-Toledo y Román-Román, 2009; GiménezToledo y otros, 2013). Colciencias por su parte, está llamada a ampliar la discusión sobre el sistema evaluativo de este tipo de formato con el fin de ofrecer garantías acerca del nivel mínimo de calidad, pertinencia y relevancia de tales contribuciones.

Por otro lado, el modelo de evaluación colombiano reconoce las tesis de maestría y doctorado como productos científicos, al considerarlas como resultados de investigación que cuentan con asesoría especializada y revisión por pares, además de ser productos finales de procesos de formación de recursos humanos para la investigación científica. La inclusión de estos trabajos como productos de los grupos de investigación conlleva un franco reconocimiento de la diversidad de formatos o formas de comunicación del conocimiento en las diferentes disciplinas de la ciencia, lo cual posiciona al modelo colombiano como uno de los que intenta mostrar suficiente apertura y visión progresista de lo que se considera producción científica (VélezCuartas y otros, 2014). Esto es particularmente relevante si se reconoce que la literatura especializada resalta el valor de las tesis como fuentes de generación de artículos en índices de alto impacto (Sánchez y otros, 2017), además de ser uno de los principales medios de uso y aprovechamiento de la producción escrita en artículos y libros (Becker y Chiware, 2015; Rosenberg, 2015).
El aumento progresivo de dirección de tesis por investigadores adscritos a grupos de investigación colombianos corresponde con la oferta que existe de programas de postgrado, así como el mayor ingreso a este nivel de formación. Cada día es más común adelantar estudios de maestría y doctorado cuya culminación se ajusta a la presentación de trabajos de investigación en el formato de tesis, las mismas se difunden a través de repositorios y diversos medios digitales que facilitan la consulta de sus contenidos, como señalan Sánchez y otros (2017), hoy más que nunca hay facilidad de acceder a trabajos de formación posgradual gracias al uso de herramientas tecnológicas, lo que conduce a un mayor consumo y apropiación de esta producción, que anteriormente se restringía al uso en bibliotecas físicas.

Precisamente, en el modelo de clasificación construido, las tesis doctorales junto con los artículos incluidos en WoS y Scopus son los productos que predicen la clasificación de los grupos de investigación. A manera de síntesis podemos reiterar algunos resultados esenciales: Los artículos incluidos en WoS y Scopus son el principal producto para la clasificación de grupos en categoría A1; para el caso de la categoría $A$, sobresale la producción de tesis doctorales $y$, finalmente, los grupos B se explican por bajos indicadores en ambos tipos de productos.

La producción analizada permite pronosticar con bastante certeza los grupos categoría B, pero los grupos categorizados en $A 1$ y $A$ no se pronostican con precisión. Adicionalmente, entre las categorías A y $B$ no se aprecian mayores diferencias pues se produce cantidad similar de libros, capítulos de libro y artículos tipo D; sólo se diferencian en la cantidad de tesis de doctorado y de artículos incluidos en WoS y Scopus, que es mayor en grupos categoría A. De acuerdo con la tipología de productos considerados en este estudio, y en función del bajo peso de los libros, capítulos de libro y tesis de maestría, vale la pena hacer un ejercicio reflexivo en torno a si resulta realmente funcional la diferenciación entre categoría A y B. Un análisis complementario que incluya la revisión de otros indicadores como el índice h del grupo, la cohesión grupal, la cooperación entre autores y la precisión de los cuartiles en que realizan sus publicaciones, podría dibujar un panorama más claro acerca de las diferencias entre categorías, por lo pronto, ajustados a la evidencia reunida en este estudio, las diferencias son mínimas.

Es muy probable que el tipo de perfil académico deseado sea el $\mathrm{A} 1$ y que éste se transforme en una exigencia laboral y de producción, al final de cuentas, para el sistema científico colombiano la máxima categorización de los grupos de investigación es sinónimo de excelencia. Acorde con nuestros hallazgos, la categorización del máximo nivel es pronosticada por una producción significativa de artícu- 
los incluidos en los índices bibliográficos de mayor prestigio e impacto mundial, esto sin duda, es la tendencia que se quiere promover con el sistema de ciencia y tecnología colombiano para incrementar los índices de producción nacional, que hasta la fecha siguen siendo bajos en comparación con países considerados potencias científicas como Estados Unidos (Shin y Cummings, 2010), o incluso frente a vecinos latinoamericanos (RICYT, 2016); así las cosas, en la medida que un grupo de investigación desee ser promovido a una categoría más elevada debe publicar más en revistas incluidas en los índices WoS y Scopus. Hay con ello una respuesta a las exigencias del "mercado académico", en cuanto la dinámica de generación de conocimiento tiende a ajustarse a los formatos más requeridos hoy día por las instituciones universitarias y organismos financiadores de la ciencia. De alguna manera se están dejando fuera otros formatos para escribir y otras metodologías de comunicación de saberes.

Colciencias viene haciendo esfuerzos por crear un nuevo sistema de medición que incluye diversas categorías de producción, sin embargo, la generación de conocimiento en relación con muchos tipos de productos es todavía rudimentaria, siendo las formas más comunes de producción aquellas que se abordaron en este estudio, y de todas ellas, sobresale la publicación de artículos en índices internacionales. Se puede estar haciendo esfuerzos metodológicos importantes cuando el verdadero valor radica en un conjunto reducido de productos dadas las características del modelo matemático que otorga elevada relevancia a esta producción. Intrínsecamente, el modelo científico colombiano promueve apertura y estrategias novedosas para reconocer lo que se asume como productos acadé- micos, pero al mismo tiempo privilegia un formato de difusión en particular que se ajusta al mercado internacional (Vélez-Cuartas y otros, 2014), o bien se está poniendo como deseable un formato de escritura y metodológico, coherente con las exigencias de las publicaciones WoS y Scopus.

\section{AGRADECIMIENTOS}

Los autores agradecen al Departamento Nacional de Ciencia y Tecnología de Colombia (Colciencias), organismo financiador del proyecto de investigación denominado "Cienciometría del campo de estudios en Ciencias Sociales en Colombia (2006-2015): Análisis de la producción científica, redes de coautoría, cooperación institucional y posicionamiento de grupos de investigación" (código SIGP01800074054754), el cual dio origen a este artículo.

También agradecen a la auxiliar de investigación Laura Rambal Rivaldo, por su contribución en el diseño, carga y organización de bases de datos.

\section{ACKNOWLEDGEMENTS}

The authors thank the National Department of Science and Technology of Colombia (Colciencias), funding agency of the research project called "Scientometrics of Social Sciences in Colombia (20062015): Analysis of scientific production, co-authorship networks, institutional cooperation and positioning of research groups" (code SIGP01800074054754), which gave rise to this article.

They also thank the research assistant Laura Rambal Rivaldo, for her contribution in the design, loading and organization of databases.

\section{REFERENCIAS}

Aldana, E. (2010). El rol de las universidades en el desarrollo científico-tecnológico: 1998-2007. Chile: Centro Interuniversitario de Desarrollo (CINDA).

Archambault, E.; Vignola-Gagne, E.; Côté, G.; Larivière, V.; Gingrasb, Y. (2006). Benchmarking scientific output in the social sciences and humanities: The limits of existing databases. Scientometrics, 68(3), 329-342. https://doi.org/10.1007/s11192-006-0115-z

Becker, D. A.; Chiware, E. R.T. (2015). Citation Analysis of Masters' Theses and Doctoral Dissertations: Balancing Library Collections with Students' Research Information Needs. The Journal of Academic Librarianship, 41(5), 613-620. https://doi.org/10.1016/j.acalib.2015.06.022

Breiman, L.; Friedman, J. H.; Olshen, R. A.; Stone, C. J. (1984). Classification and Regression Trees. Belmont, CA, EEUU.: Wadsworth International Group.

Bucheli, V.; Díaz, A.; Calderón, J.; Lemoine, P.; Valdivia, J.; Villaveces, J.; Zarama, R. (2012). Growth of scientific production in Colombian universities: an intellectual capital-based approach. Scientometrics, 91(2), 369382. https://doi.org/10.1007/s11192-012-0627-7

Colciencias - Departamento Administrativo de Ciencia, Tecnología e Innovación (2014). Modelo de medición de Grupos de Investigación, Desarrollo Tecnológico o de Innovación y de reconocimiento de investigadores del Sistema Nacional de Ciencia, Tecnología e Innovación, año 2014. Bogotá D.C.: Dirección de Fomento a la Investigación.

Colciencias - Departamento Administrativo de Ciencia, Tecnología e Innovación (2015). El Estado de la Ciencias en Colombia. Disponible en: https://sites.google. com/a/colciencias.gov.co/estado-de-la-ciencia-2015/ [Consultado 23/02/2017]. 
Colciencias - Departamento Administrativo de Ciencia, Tecnología e Innovación. (2017). Modelo de medición de grupos de investigación, desarrollo tecnológico o de innovación y reconocimiento de investigadores del sistema nacional de ciencia tecnología e innovación año 2017. Disponible en: https://goo.gl/COU4ey [Consultado 10/09/2017].

Daud, A.; Ahmad, M.; Malik, M.S.I.; Che, D. (2015). Using machine learning techniques for rising star prediction in co-author network. Scientometrics, 102(2), 16871711. https://doi.org/10.1007/s11192-014-1455-8

Fernández-Cano, A.; Torralbo, M.; Vallejo, M. (2011). Time series of scientific growth in Spanish doctoral theses (1848-2009). Scientometrics, 91(1), 15-36. https://doi.org/10.1007/s11192-011-0572-x

Gantman, E. R. (2011). La productividad científica argentina en Ciencias Sociales: Economía, Psicología, Sociología y Ciencia Política en el CONICET (2004-2008). Revista Española de Documentación Científica, 34(3), 408-425. https://doi.org/10.3989/redc.2011.3.829

Giménez-Toledo, E.; Román-Román, A. (2009). Assessment of humanities and social sciences monographs through their publishers: A review and a study towards a model of evaluation. Research Evaluation, 18(3), 201-213. https://doi.org/10.3152/095820209X471986

Giménez-Toledo, E., Tejada-Artigas, C.; Manana-Rodriguez, J. (2013). Evaluation of scientific books' publishers in social sciences and humanities: Results of a survey. Research Evaluation, 22(1), 64-77. https:// doi.org/10.1093/reseval/rvs036

Hicks, D. (2004). The four literatures of social science. En: Handbook of quantitative science and technology research, pp 473-496. Kluber Academic.

Huang, M.H. ; Lin, C.S. (2011). A citation analysis of Western journals cited in Taiwan's Library and Information Science and History research journals: From a research evaluation perspective. Journal of Academic Librarianship, 37(1), 34-45. https://doi.org/10.1016/j. acalib.2010.10.005

Ibarra, A. ; Castro, J.; Barrenechea, J. (Eds. ). (2006). La evaluación de la actividad científica en Ciencias Sociales y Humanidades. Servicio editorial de la Universidad del País Vasco. Euskal Herriko Unibertsitatea Argitalpen Zerbitua.

Larivière, V.; Sugimoto, C.R.; Cronin, B. (2012). A bibliometric chronicling of Library and Information Science's first hundred years. Journal of the American Society for Information Science and Technology, 63(5), 9971016. https://doi.org/10.1002/asi.22645

Low, Y.; Ng, K. H.; Kabir, M. A.; Koh, A. P.; Sinnasamy, J. (2014). Trend and impact of international collaboration in clinical medicine papers published in Malaysia. Scientometrics, 98(2), 1521-1533. https://doi. org/10.1007/s11192-013-1121-6

Nederhof, A. (2006). Bibliometric monitoring of research performance in the Social Sciences and the Humanities: A Review. Scientometrics, 66(1), 81-100. https:// doi.org/10.1007/s11192-006-0007-2
OCyT - Observatorio Colombiano de Ciencia y Tecnología (2016). Indicadores de ciencia y tecnología. Bogotá, Colombia.

RICYT - Red de Indicadores de Ciencia y Tecnología Iberoamericana e Interamericana- (2015). Indicadores Bibliométricos. Disponible en http://www.ricyt.org/ comparativos-sp-469065143 [consultado 30/07/2017].

RICYT - Red de Indicadores de Ciencia y Tecnología Iberoamericana e Interamericana- (2016). Estado de la ciencia en Colombia. Bogotá: Colciencias.

Rosario-Sierra, M.; Sánchez-Perdomo, R.; Herrera-Vallejera, D.; Rodríguez-Sánchez, Y. (2017). Patrón de citas de la producción científica en Ciencias Sociales y Humanidades. Un análisis a partir de Scopus (20002012). Ciencias de la Información, 48(1), 37-44.

Rosenberg, Z. (2015). Citation Analysis of M.A. Theses and Ph.D. Dissertations in Sociology and Anthropology: An Assessment of Library Resource Usage. The Journal of Academic Librarianship, 41(5), 680-688. https://doi.org/10.1016/j.acalib.2015.05.010

Rueda-Barrios, G.; Rodenes-Adam, M. (2016). Factores determinantes en la producción científica de los grupos de investigación en Colombia. Revista Española de Documentación Científica, 39(1): e118. https://doi. org/10.3989/redc.2016.1.1198

Sánchez, R.; Blázquez, M.; Montesi, M.; Botezan, I. (2017). La producción de tesis doctorales en España (1995-2014): evolución, disciplinas, principales actores y comparación con la producción científica en WoS y Scopus. Revista Española de Documentación Científica, 40(4): e188. https://doi.org/10.3989/ redc. 2017.4.1409

Sanz-Casado, E. ; Filippo, D. de; García Zorita, C.; EfraínGarcía, P. (2011). Observatorio IUNE: una nueva herramienta para el seguimiento de la actividad investigadora del sistema universitario español. Bordón. Revista de Pedagogía, 63(2), 101-116.

Shin, J.; Cummings, W. (2010). Multilevel analysis of academic publishing across disciplines: research preference, collaboration, and time on research. Scientometrics, 85(2), 581-594. https://doi.org/10.1007/ s11192-010-0236-2

Steinberg, D. (2009). CART: Classification and Regression Trees. En: Wu, X.; Kumar, V. (eds.), The top ten algorithms in data mining. Boca Raton, FL.; USA: Chapman \& Hall/CRC Taylor \& Francis Group.

Thompson, J. B. (2005). Books in the digital age. The Transformation of academic and higher education publishing in Britain and the United States. Cambridge, UK: Polity.

Vélez-Cuartas, G.; Gómez-Flórez, H.; Úsuga-Ciro, A.; Vélez-Trujillo, M. (2014). Diversidad y reconocimiento de la producción académica en los sistemas de evaluación de la investigación en Colombia. Revista Española de Documentación Científica, 37(3): e056. https://doi. org/10.3989/redc.2014.3.1133 
Vélez-Cuartas, G.; Lucio-Arias, D.; Leydesdorff, L. (2016). Regional and global science: Publications from Latin America and the Caribbean in the SciELO Citation Index and the Web of Science. El Profesional de la Información, 25(1): 35-46. https://doi.org/10.3145/epi.2016.ene.05

Verleysen, F. T.; Engels, T. C. E. (2014). Barycenter representation of book publishing internationalization in the Social Sciences and Humanities. Journal of Informetrics 8(1), 234-240. https://doi.org/10.1016/j. joi.2013.11.008

Vessuri, H.; Sonsiré, M. (2010). Institutional aspects of the Social Sciences in Latin America. En: World Social Science Report. Knowledge Divides. (pp. 59-62). Paris: International Social Science Council and UNESCO. 